\title{
$5-14-1998$
}

\section{Shape Invariance and Its Connection to Potential Algebra}

Asim Gangopadhyaya

Loyola University Chicago, agangop@luc.edu

Jeffrey Mallow

Loyola University Chicago, jmallow@luc.edu

Uday P. Sukhatme

University of Illinois at Chicago, sukhatme@uic.edu

Follow this and additional works at: https://ecommons.luc.edu/physics_facpubs

Part of the Quantum Physics Commons

\section{Author Manuscript}

This is a pre-publication author manuscript of the final, published article.

\section{Recommended Citation}

Gangopadhyaya, A, J Mallow, and U Sukhatme. "Shape invariance and its connection to potential algebra." http://arxiv.org/pdf/quant-ph/9805042.pdf

This Presentation is brought to you for free and open access by the Faculty Publications and Other Works by Department at Loyola eCommons. It has been accepted for inclusion in Physics: Faculty Publications and Other Works by an authorized administrator of Loyola eCommons. For more information, please contact ecommons@luc.edu.

\section{c) (†) $\ominus$}

This work is licensed under a Creative Commons Attribution-Noncommercial-No Derivative Works 3.0 License. (C) 1998 The Authors. 
February 1, 2008

\title{
Shape Invariance and Its Connection to Potential Algebra
}

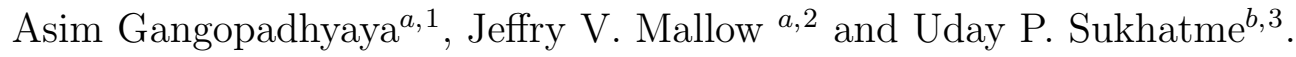 \\ a) Department of Physics, Loyola University Chicago, Chicago, IL 60626; \\ b) Department of Physics, University of Illinois at Chicago, (m/c 273) \\ 845 W. Taylor Street, Chicago, IL 60607-7059.
}

\begin{abstract}
Exactly solvable potentials of nonrelativistic quantum mechanics are known to be shape invariant. For these potentials, eigenvalues and eigenvectors can be derived using well known methods of supersymmetric quantum mechanics. The majority of these potentials have also been shown to possess a potential algebra, and hence are also solvable by group theoretical techniques. In this paper, for a subset of solvable problems, we establish a connection between the two methods and show that they are indeed equivalent.
\end{abstract}

\footnotetext{
${ }^{1}$ e-mail: agangop@luc.edu, asim@uic.edu

${ }^{2}$ e-mail: jmallow@luc.edu

${ }^{3}$ e-mail: sukhatme@uic.edu
} 


\section{Introduction}

It is well known that most of the exactly solvable potentials of nonrelativistic quantum mechanics fall under the Natanzon class (酒) where the Schrödinger equation reduces either to the hypergeometric or the confluent hypergeometric differential equations. A few exceptions are known ([2, 3]), where solvable potentials are given as a series, and can not be written in closed form in general. With the exception of Ginnochio potential, all exactly solvable potentials are known to be shape invariant ([4, 5]); i.e. their supersymmetric partners are of the same shape, and their spectra can be determined entirely by an algebraic procedure, akin to that of the one dimensional harmonic oscillator, without ever referring to the underlying differential equations $([6])$.

Several of these exactly solvable systems are also known to possess what is generally referred to as a potential algebra $([7,8,9,10,12$, 11]). The Hamiltonian of these systems can be written as the Casimir of an underlying $\mathrm{SO}(2,1)$ algebra, and all the quantum states of these systems can be determined by group theoretical methods.

Thus, there appear to be two seemingly independent algebraic methods for obtaining the complete spectrum of these Hamiltonians. In this paper, we analyze this ostensible coincidence. For a category of solvable potentials, we find that these two approaches are indeed related.

In the next section, we briefly describe supersymmetric quantum mechanics (SUSYQM), and discuss how the constraint of shape invariance suffices to determine the spectrum of a shape invariant potential (SIP). In sec. 3, we judiciously construct some algebraic operators and show that the shape invariance constraint can be expressed as an algebraic condition. For a set of shape invariant potentials, we find that the shape invariance condition leads to the presence of a $\mathrm{SO}(2,1)$ potential algebra, and we thus establish a connection between the two algebraic methods. In sec. 4, for completeness, we provide a brief review of $\mathrm{SO}(2,1)$ representation theory. In sec. 5, we derive the spectrum of a class of potentials and explicitly show that both methods indeed give identical spectrum. 


\section{SUSY-QM and Shape Invariance}

A quantum mechanical system specified by a potential $V_{-}(x)$ can alternatively be described by its ground state wavefunction $\psi_{0}^{(-)}$. Apart from a constant (chosen suitably to make the ground state energy zero), it follows from the Schrödinger equation that the potential can be written as $V_{-}(x)=\left(\frac{\psi_{0}^{\prime \prime}}{\psi_{0}}\right)$, where prime denotes differentiation with respect to $x$. In SUSY-QM, it is customary to express the system in terms of the superpotential $W(x)=-\left(\frac{\psi_{0}^{\prime}}{\psi_{0}}\right)$ rather than the potential, and the ground state wavefunction is then given by $\psi_{0} \sim \exp \left(-\int_{x_{0}}^{x} W(x) d x\right)$, where $x_{0}$ is an arbitrarily chosen reference point. We are using units with $\hbar$ and $2 m=1$. The Hamiltonian $H_{-}$can now be written as

$$
H_{-}=\left(-\frac{d^{2}}{d x^{2}}+V_{-}(x)\right)=\left(-\frac{d^{2}}{d x^{2}}+W^{2}(x)-\frac{d W(x)}{d x}\right) \text {. }
$$

However, as we shall see, there is another Hamiltonian $H_{+}$with potential $V_{+}(x)=\left(W^{2}(x)+\frac{d W(x)}{d x}\right)$, that is almost iso-spectral with the original potential $V_{-}(x)$. In particular, the eigenvalues $E_{n}^{+}$of $H_{+}(x)$ satisfy $E_{n}^{+}=E_{n+1}^{-}$, where $E_{n}^{-}$are eigenvalues of $H_{-}(x)$ and $n=0,1,2, \cdots$, i.e. except the ground state all other states of $H_{-}$are in one-to-one correspondence with states of $H_{+}$. The potentials $V_{-}(x)$ and $V_{+}(x)$ are known as supersymmetric partners.

In analogy with the harmonic oscillator, we now define two operators: $A \equiv\left(\frac{d}{d x}+W(x)\right)$, and and its Hermitian conjugate $A^{+} \equiv\left(-\frac{d}{d x}+W(x)\right)$. Hamiltonians $H_{-}$and its superpartner $H_{+}$are given by operators $A^{+} A$ and $A A^{+}$respectively.

Now we shall explicitly establish the iso-spectral relationship between states of $H_{+}$and $H_{-}$. Let us denote the eigenfunctions of $H_{ \pm}$that correspond to eigenvalues $E_{n}^{ \pm}$, by $\psi_{n}^{( \pm)}$. For $n=1,2, \cdots$,

$$
\begin{aligned}
H_{+}\left(A \psi_{n}^{(-)}\right) & =A A^{+}\left(A \psi_{n}^{(-)}\right)=A\left(A^{+} A \psi_{n}^{(-)}\right)=A H_{-}\left(\psi_{n}^{(-)}\right) \\
& =E_{n}^{-}\left(A \psi_{n}^{(-)}\right) .
\end{aligned}
$$

Hence, excepting the ground state which obeys $A \psi_{0}^{(-)}=0$, for any state $\psi_{n}^{(-)}$of $H_{-}$ there exists a state $A \psi_{n}^{(-)}$of $H_{+}$with exactly the same energy, i.e. $E_{n-1}^{+}=E_{n}^{-}$, where $n=1,2, \cdots$, i.e. $A \psi_{n}^{(-)} \propto \psi_{n-1}^{(+)}$. Conversely, one also has $A^{+} \psi_{n}^{(+)} \propto \psi_{n+1}^{(-)}$. Thus, if the eigenvalues and the eigenfunctions of $H_{-}$were known, one would automatically obtain 
the eigenvalues and the eigenfunctions of $H_{+}$, which is in general a completely different Hamiltonian.

Now, let us consider the special case where $V_{-}(x)$ is a SIP. This implies that $V_{-}(x)$ and $V_{+}(x)$ have the same functional form; they only differ in values of other discrete parameters and possibly an additive constant. To be explicit, let us assume that in addition to the continuous variable $x$, the potential $V_{-}(x)$ also depends upon a constant parameter $a_{0}$; i.e., $V_{-} \equiv V_{-}\left(x, a_{0}\right)$. The ground state of the system of $H_{-}$is given by $\psi_{0}\left(x, a_{0}\right) \sim \exp \left(-\int_{x_{0}}^{x} W\left(x, a_{0}\right) d x\right)$. Now, for a shape invariant $V_{-}\left(x, a_{0}\right)$, one has, $V_{+}\left(x, a_{0}\right)=V_{-}\left(x, a_{1}\right)+R\left(a_{0}\right)$, where $R\left(a_{0}\right)$ is the additive constant mentioned above. Since potentials $V_{+}\left(x, a_{0}\right)$ and $V_{-}\left(x, a_{1}\right)$ differ only by $R\left(a_{0}\right)$, their common ground state is given by $\psi_{0}\left(x, a_{1}\right) \sim \exp \left(-\int_{x_{0}}^{x} W\left(x, a_{1}\right) d x\right)$. Now using SUSY-QM algebra, the first excited state of $H_{-}\left(x, a_{0}\right)$ is given by $A^{+}\left(x, a_{0}\right) \psi_{0}^{(-)}\left(x, a_{1}\right)$. Its energy is $E_{1}^{(-)}$, which is equal to $E_{0}^{(+)}$. But since $E_{0}^{(-)}=0, E_{0}^{(+)}$must be $R\left(a_{0}\right)$. Continuing up the ladder of series of potentials $V_{-}\left(x, a_{i}\right)$, we can obtain the entire spectrum of $H_{-}$by algebraic methods of SUSY-QM. The eigenvalues are given by

$$
E_{0}^{(-)}=0 ; \quad \text { and } \quad E_{n}^{(-)}=\sum_{k=0}^{n-1} R\left(a_{k}\right) \text { for } n>0
$$

and the $n$-th eigenstate is given by

$$
\psi_{n+1}^{(-)}\left(x, a_{0}\right) \sim A^{+}\left(a_{0}\right) A^{+}\left(a_{1}\right) \cdots A^{+}\left(a_{n-1}\right) \psi_{0}^{(-)}\left(x, a_{n-1}\right)
$$

(To avoid notational complexity, we have suppressed the $x$-dependence of operators $A\left(x, a_{0}\right)$ and $A^{+}\left(x, a_{0}\right)$.)

\section{Shape Invariance and Potential Algebra}

Let us consider the special case of a potential $V_{-}\left(x, a_{0}\right)$ with an additive shape invariance; i.e. $V_{+}\left(x, a_{0}\right)=V_{-}\left(x, a_{1}\right)+R\left(a_{0}\right)$, where $a_{n}=a_{n-1}+\delta=a_{0}+n \delta$, where $\delta$ is a constant. Most SIP's fall into this category. For the superpotential $W\left(x, a_{m}\right) \equiv W(x, m)$, the shape invariance condition implies

$$
W^{2}(x, m)+W^{\prime}(x, m)=W^{2}(x, m+1)-W^{\prime}(x, m+1)+R(m)
$$

As described in the last section, this constraint suffices to determine the entire spectrum 
of the potential $V_{-}(x, m)$. In this section, we shall explore the possible connection of this method with the potential algebra discussed by several authors $([0,8,9,10,12,11])$.

Since for a SIP, the parameter $m$ is changed by a constant amount each time as one goes from the potential $V_{-}(x, m)$ to its superpartner, it is natural to ask whether such a task can be formally accomplished by the action of a ladder-type operator.

With that in mind, we first define an operator $J_{3}=-i \frac{\partial}{\partial \phi}$, analogous to the $z$-component of the angular momentum operator. It acts upon functions in the space described by two coordinates $x$ and $\phi$, and its eigenvalues $m$ play the role of the parameter of the potential. We also define two more operators, $J^{-}$and its Hermitian conjugate $J^{+}$by

$$
J^{ \pm}=e^{ \pm i \phi}\left[ \pm \frac{\partial}{\partial x}-W\left(x,-i \frac{\partial}{\partial \phi} \pm \frac{1}{2}\right)\right]
$$

The factors $e^{ \pm i \phi}$ in $J^{ \pm}$ensure that they indeed operate as ladder operators for the quantum number $m$. Operators $J^{ \pm}$are basically of the same form as the $A^{ \pm}$operators described earlier in sec. 2, except that the parameter $m$ of the superpotential is replaced by operators $\left(J_{3} \pm \frac{1}{2}\right)$. With explicit computation we find

$$
\left[J_{3}, J^{ \pm}\right]= \pm J^{ \pm}
$$

and hence operators $J^{ \pm}$change the eigenvalues of the $J_{3}$ operator by unity, similar to the ladder operators of angular momentum $(S U(2))$. Now let us determine the remaining commutator $\left[J^{+}, J^{-}\right]$. The product $J^{+} J^{-}$is given by

$$
\begin{aligned}
J^{+} J^{-} & =e^{i \phi}\left[\frac{\partial}{\partial x}-W\left(x, J_{3}+\frac{1}{2}\right)\right] e^{-i \phi}\left[-\frac{\partial}{\partial x}-W\left(x, J_{3}-\frac{1}{2}\right)\right] \\
& =\left[-\frac{\partial^{2}}{\partial x^{2}}+W^{2}\left(x, J_{3}-\frac{1}{2}\right)-W^{\prime}\left(x, J_{3}-\frac{1}{2}\right)\right]
\end{aligned}
$$

Similarly,

$$
J^{-} J^{+}=\left[-\frac{\partial^{2}}{\partial x^{2}}+W^{2}\left(x, J_{3}+\frac{1}{2}\right)+W^{\prime}\left(x, J_{3}+\frac{1}{2}\right)\right] .
$$

Hence the commutator of operators $J_{+}$and $J_{-}$is given by

$$
\begin{aligned}
{\left[J^{+}, J^{-}\right]=} & {\left[-\frac{\partial^{2}}{\partial x^{2}}+W^{2}\left(x, J_{3}-\frac{1}{2}\right)-W^{\prime}\left(x, J_{3}-\frac{1}{2}\right)\right] } \\
& -\left[-\frac{\partial^{2}}{\partial x^{2}}+W^{2}\left(x, J_{3}+\frac{1}{2}\right)+W^{\prime}\left(x, J_{3}+\frac{1}{2}\right)\right] \\
= & -R\left(J_{3}+\frac{1}{2}\right),
\end{aligned}
$$


where we have used the constraint of shape invariance, i.e. $V_{-}\left(x, J_{3}-\frac{1}{2}\right)-V_{+}\left(x, J_{3}+\frac{1}{2}\right)=$ $-R\left(J_{3}+\frac{1}{2}\right)$. Thus, we see that Shape Invariance enables us to close the algebra of $J_{3}$ and $J^{ \pm}$to

$$
\left[J_{3}, J^{ \pm}\right]= \pm J^{ \pm}, \quad\left[J^{+}, J^{-}\right]=-R\left(J_{3}+\frac{1}{2}\right)
$$

Now, if the function $R\left(J_{3}\right)$ were linear in $J_{3}$, the algebra of eq.(95) would reduce to that of a $\mathrm{SO}(3)$ or $\mathrm{SO}(2,1)$. Several SIP's are of this type, among them are the Morse, the Rosen-

Morse and the Pöschl-Teller I and II potentials. For these potentials, $R\left(J_{3}+\frac{1}{2}\right)=2 J_{3}$, and eq.(9) reduces to an $\mathrm{SO}(2,1)$ algebra and thus establishes the connection between shape invariance and potential algebra. Even though there is much similarity between $\mathrm{SO}(2,1)$ and $\mathrm{SO}(3)$ algebras, there are some important differences between their representations. Hence, for completeness, we will briefly describe the unitary representations of $\mathrm{SO}(2,1)$ and refer the reader to [13] for a more detailed presentation.

\section{Unitary Representations of $\mathrm{SO}(2,1)$ Algebra}

In this section, we shall briefly review the $\mathrm{SO}(2,1)$ algebra and its unitary representations (unireps). This description is primarily based upon a review article by B.G. Adams, J. Cizeka and J. Paldus (1987). The generators of the SO(2,1) algebra satisfy

$$
\left[J_{3}, J^{ \pm}\right]= \pm J^{ \pm} ;\left[J_{+}, J_{-}\right]=-2 J_{3}
$$

where $J^{ \pm}$are related to their Cartessian counterparts by $J^{ \pm}=J_{1} \pm J_{2}$. (For the familiar $\mathrm{SO}(3)$ case, one has $\left.\left[J_{+}, J_{-}\right]=+2 J_{3}\right)$. The Casimir of the $\mathrm{SO}(2,1)$ algebra is

$$
J^{2}=-J^{+} J^{-}+J_{3}^{2}-J_{3}=-J^{-} J^{+}+J_{3}^{2}+J_{3}
$$

In analogy to the representation of angular momentum algebra, one can choose $J^{2}$ and one of the $J_{i}$ 's as two commuting observables. However, unlike the $\mathrm{SO}(3)$ case, each such choice of a pair generates a different set of inequivalent representations. For bound states, we choose the familiar representation space of states $|j, m\rangle$ on which the operators $\left\{J^{2}, J_{3}\right\}$ are diagonal: $J^{2}|j, m\rangle=j(j+1)|j, m\rangle, J_{3}|j, m\rangle=m|j, m\rangle$. Operators $J^{ \pm}$act upon $|j, m\rangle$ states as ladder operators: $J^{ \pm}|j, m\rangle=[-(j \mp m)(j \pm m+1)]^{\frac{1}{2}}|j, m+1\rangle$. Since the quantum number $m$ increases in unit steps for a given $j$, the general value for $m$ is of the form 
$m_{0}+n$, where $n$ is an integer and $m_{0}$ is a real number. There is also another constraint on the quantum numbers $m$ and $j$. In unitary representations, $J^{+}$and $J^{-}$are Hermitian conjugates of each other, and $J^{+} J^{-}$and $J^{-} J^{+}$are therefore positive operators. This implies $[-(j \mp m)(j \pm m+1)]=-\left[\left(j+\frac{1}{2}\right)^{2}-\left(m+\frac{1}{2}\right)^{2}\right] \geq 0$. These constraints can be illustrated on a two dimensional planar diagram [Fig. 1] depicting the allowed values of $m$ and $j$. Only the open triangular areas DFB, HEG and the square AEFC are the allowed regions. The values of $|m|$ are no longer bounded by $j$, and depending on the $m_{0}$ (the starting value of $m$ ), representations multiplets are either semi-infinite (bounded from below or above) or completely unbounded. Thus there is no finite (nontrivial) unitary representation of $\mathrm{SO}(2,1)$. In general, there are four classes of unireps.

$$
\begin{aligned}
& \begin{array}{l}
\text { Bounded from below } \\
\left(j, m_{0}\right) \text { lie along } \\
\text { the segment AB }
\end{array} \quad\left\{\begin{array}{l}
m=-j+n ; n=0,1,2, \cdots, \\
j<0,
\end{array}\right.
\end{aligned}
$$

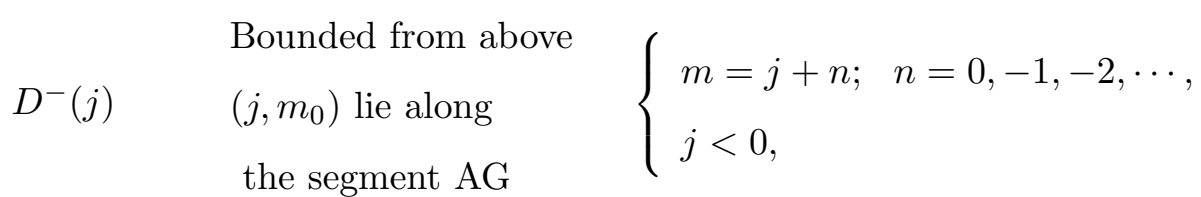

$$
\begin{aligned}
& \begin{array}{l}
\left(j, m_{0}\right) \text { lie in } \\
\text { the square area }
\end{array} \quad\left\{\begin{array}{l}
m=m_{0}+n ; n=0, \pm 1, \pm 2, \cdots \\
j(j+1)<\left(\left|m_{0}\right|-1\right)\left|m_{0}\right| \\
-\frac{1}{2}<m_{0}<-\frac{1}{2}
\end{array}\right. \\
& D_{p}\left(j, m_{0}\right) \quad \begin{array}{l}
\text { Unbounded and } \\
\text { complex } j
\end{array} \quad\left\{\begin{array}{l}
m=m_{0}+n ; n=0, \pm 1, \pm 2, \cdots \\
-\frac{1}{2}<m_{0}<-\frac{1}{2} \\
j=-\frac{1}{2}+i \beta
\end{array}\right.
\end{aligned}
$$

Here we will be interested in representations that are bounded from either below or above. Such representations fall in triangular areas DFB and HEG.

For the $D^{+}$representation, the starting value of $m$ can be anywhere on the darkened part of the line AB; other allowed values of $m$ are then obtained by the action of the ladder operator $J^{+}$. Owing to the equivalence of $D^{+}(j)$ and $D^{+}(-j-1)$, they correspond to the 
same value of $j(j+1)$. One could have equivalently started anywhere on the segment $\mathrm{CD}$ as well and used $D^{+}(-j-1)$. Both are equivalent and each is unique. Similarly, for complete $D^{-}(j)\left(D^{-}(-j-1)\right)$ representation, one starts from AG $(\mathrm{GH})$ and generates all other states by the action of the $J^{-}$operator.

\section{Example}

As a concrete example, we will examine the Scarf potential which can be related to the Pöschl-Teller II potential by a redefinition of the independent variable. We will show that the shape invariance of the Scarf potential automatically leads to its potential algebra: $\mathrm{SO}(2,1)$. (Exactly similar analysis can be carried out for the Morse, the Rosen-Morse, and the Pöschl-Teller potentials.) The Scarf potential is described by its superpotential $W\left(x, a_{0}, B\right)=a_{0} \tanh x+B \operatorname{sech} x$. The potential $V_{-}\left(x, a_{0}, B\right)=W^{2}\left(x, a_{0}, B\right)-W^{\prime}\left(x, a_{0}, B\right)$ is then given by

$$
V_{-}\left(x, a_{0}, B\right)=\left[B^{2}-a_{0}\left(a_{0}+1\right)\right] \operatorname{sech}^{2} x+B\left(2 a_{0}+1\right) \operatorname{sech} x \tanh x+a_{0}^{2}
$$

The eigenvalues of this system are given by $([6])$

$$
E_{n}=a_{0}^{2}-\left(a_{0}-n\right)^{2}
$$

The partner potential $V_{+}\left(x, a_{0}, B\right)=W^{2}\left(x, a_{0}, B\right)+W^{\prime}\left(x, a_{0}, B\right)$ is given by

$$
\begin{aligned}
V_{+}\left(x, a_{0}, B\right) & =\left[B^{2}-a_{0}\left(a_{0}-1\right)\right] \operatorname{sech}^{2} x+B\left(2 a_{0}-1\right) \operatorname{sech} x \tanh x+a_{0}^{2} . \\
& =V_{-}\left(x, a_{1}, B\right)+a_{0}^{2}-a_{1}^{2}
\end{aligned}
$$

where $a_{1}=a_{0}-1$. Thus, $R\left(a_{0}\right)$ for this case is $a_{0}^{2}-a_{1}^{2}=2 a_{0}-1$, linear in $a_{0}$.

Now, following the mechanism of the sec. 2, consider a set of operators $J^{ \pm}$which is given by

$$
J^{ \pm}=e^{ \pm i \phi}\left[ \pm \frac{\partial}{\partial x}-\left\{\left(-i \frac{\partial}{\partial \phi} \pm \frac{1}{2}\right) \tanh x+B \operatorname{sech} x\right\}\right] .
$$

Note the similarity between the operators $J^{ \pm}$and operators $A^{ \pm}$defined in sec. 2. Since only the parameter $a_{0}$ changes in the shape invariance condition, it is replaced by $J_{3} \pm \frac{1}{2}$. Commutators of these operators with $J_{3}=-i \frac{\partial}{\partial \phi}$ can be shown to close on $J^{ \pm}$, as discussed in general in Sec. 2. Now, from eq.(9) and (14), the commutator of $J^{ \pm}$operators is given 
by $-2 J_{3}$, thus forming a closed $\mathrm{SO}(2,1)$ algebra. Moreover, the operator $J^{+} J^{-}$, acting on the basis $|j, m\rangle$ gives:

$$
\begin{aligned}
J^{+} J^{-} \equiv & {\left[B^{2}-\left(m^{2}-\frac{1}{4}\right)\right] \operatorname{sech}^{2} x } \\
& +B\left(2\left(m-\frac{1}{2}\right)+1\right) \operatorname{sech} x \tanh x+\left(m-\frac{1}{2}\right)^{2} .
\end{aligned}
$$

which is just the $H_{\text {scarf }}\left(x, m-\frac{1}{2}, B\right)$, i.e. the Scarf Hamiltonian with $a_{0}$ replaced by $m-\frac{1}{2}$. Thus the energy eigenvalues of the Hamiltonian will be the same as that of the operator $J^{+} J^{-}=J_{3}^{2}-J_{3}-J^{2}$. Hence, the energy is given by $E=m^{2}-m-j(j+1)$. Substituting $j=n-m$, one gets

$$
\begin{aligned}
E_{n} & =m^{2}-n-(n-m)^{2} \\
& =\left(m-\frac{1}{2}\right)^{2}-\left[n-\left(m-\frac{1}{2}\right)\right]^{2}
\end{aligned}
$$

which is the same as eq.(13), with $a_{0}$ replaced by $\left(m-\frac{1}{2}\right)$. Thus for this potential, as well as for the other three potentials mentioned above, there are actually an infinite number of potentials characterised by all allowed values of the parameter $m$ that correspond to the same value of $j$ and hence to the same energy E. Hence the name "potential algebra" $([7,12])$.

Conclusion: The algebra of Shape Invariance plays an important role in the solvability of most exactly solvable problems in quantum mechanics. Their spectrum can be easily generated simply by algebraic means. Many of these systems also have been shown to possess a potential algebra, which provides an alternate algebraic method to determine the eigenvalues and eigenfunctions. An obvious question is whether these are two unrelated algebraic methods or there is a link between them. For a subset of exactly solvable potentials, those with $R\left(a_{0}\right)$ linear in parameter $a_{0}$, we have shown the equivalence of their shape invariance property to an $\mathrm{SO}(2,1)$ potential algebra. As a concrete example, we started with the Scarf potential and showed explicitly how shape invariance translates into the $\mathrm{SO}(2,1)$ potential algebra. We determined the spectra using the algebra of $\mathrm{SO}(2,1)$ and showed them to be the same as that obtained from shape invariance.

However, we only worked with solvable models for which $R\left(J_{3}\right)$ is a linear function of $J_{3}$. There are many systems for which the above is not true. Also there were new Shape 
Invariant problems discovered in 1992 ([沟) for which it is not possible to write the potential in closed form. It will be interesting to know whether there are potential algebras that describe these system, and whether they are connected to their Shape Invariance. These are open problems and are currently under investigation.

One of us (AG) would like to thank the Physics Department of the University of Illinois for warm hospitality. We would also like to thank Dr. Prsanta Panigrahi for many related discussion.

\section{References}

[1] Natanzon, G. A. (1979): Teor. Mat. Fiz. 38, 219.

[2] Spiridonov, V. P. (1992): Phys. Rev. Lett. 69, 298.

[3] Barclay, D., Dutt, R., Gangopadhyaya, A., Khare, A., Pagnamenta, A. and Sukhatme, U. (1993): Phys. Rev. A48 2786.

[4] Gendenshtein, L. E. (1983): JETP Lett. 38, 356;

[5] Gendenshtein, L. E., Krive, I. V. (1985): Sov. Phys. Usp. 28, 645.

[6] Cooper, F., Khare, A., and Sukhatme, U. (1995): Phys. Rep. 251, 268 and references therein.

[7] Alhassid, Y., Gürsey, F. and Iachello, F. (1983): Ann. Phys. 148, 346.

[8] Barut, A.O., Inomata, A., Wilson, R. (1987): J. Phys. A: Math. Gen. 20 4075; J. Phys. A: Math. Gen. 204083.

[9] Englefield, M.J., Quesne, C. (1987): J. Phys. A: Math. Gen. 24 827(1987).

[10] Englefield, M.J. (1987): J. Phys. A: Math. Gen. 283557.

[11] Tangerman R.D., Tjon, J.A. (1993): Phys. Rev. A 481089.

[12] Wu J., Alhassid, Y. (1990): Phys. Rev. A 31557. 
[13] Adams, B.G, Cizeka, J., Paldus, J. (1987): Lie Algebraic Methods And Their Applications to Simple Quantum Systems. (From the Advances in Quantum Chemistry, Vol. 19, Edited by Per-Olov Löwdin. Academic Press). 


\section{FIGURE CAPTION:}

FIG 1. Two dimension plot showing the allowed region for $m$ and $j$. 